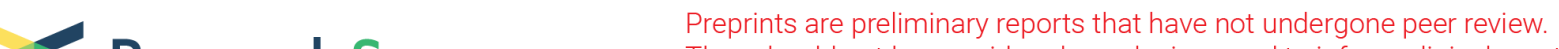 Research Square
They should not be considered conclusive, used to inform clinical practice,
or referenced by the media as validated information.
}

\section{Global heating of Jupiter's upper atmosphere by auroral energy circulation}

James O'Donoghue ( $\square$ jameso@ac.jaxa.jp )

JAXA Institute of Space and Astronautical Science https://orcid.org/0000-0002-4218-1191

\section{Luke Moore}

Boston University

\section{Tanapat Bhakyapaibul}

Boston University

\section{Henrik Melin}

University of Leicester

\section{Tom Stallard}

University of Leicester

John Connerney

NASA Goddard Space Flight Center

Chihiro Tao

National Institute of Information and Communications Technology (NICT)

\section{Physical Sciences - Article}

Keywords: Giant Planet, Magnetosphere-atmosphere Coupling Processes, Global Circulation Models, Global Temperature Gradients

Posted Date: March 2nd, 2021

DOI: https://doi.org/10.21203/rs.3.rs-275557/v1

License: (c) (1) This work is licensed under a Creative Commons Attribution 4.0 International License. Read Full License

Version of Record: A version of this preprint was published at Nature on August 4th, 2021. See the published version at https://doi.org/10.1038/s41586-021-03706-w. 


\section{Global heating of Jupiter's upper atmosphere by auroral energy circulation}

J. O’Donoghue, ${ }^{1,2 *}$ L. Moore, ${ }^{3}$ T. Bhakyapaibul ${ }^{3}$, H. Melin ${ }^{4}$,

T. Stallard ${ }^{4}$, J. E. P. Connerney ${ }^{5,2}$, C. Tao $^{6}$

${ }^{1}$ Department of Solar System Science, JAXA Institute of Space and Astronautical Science, Sagamihara city, Kanagawa Prefecture, Japan

${ }^{2}$ NASA Goddard Space Flight Center, Greenbelt, MD 20771, USA

${ }^{3}$ Center for Space Physics, Boston University, Boston, Massachusetts, USA

${ }^{4}$ Department of Physics and Astronomy, University of Leicester, Leicester, UK

${ }^{5}$ Space Research Corporation, Annapolis, MD 21403, USA

${ }^{6}$ National Institute of Information and Communications Technology (NICT), Tokyo, Japan

*E-mail: jameso@ac.jaxa.jp.

Giant planet upper atmospheres have long been observed to be significantly hotter than expected $^{1-4}$. Magnetosphere-atmosphere coupling processes give rise to auroral emissions and enormous energy deposition near the magnetic poles, explaining high temperatures for narrow regions of the planet. However, global circulation models have difficulty redistributing auroral energy globally due to the strong Coriolis forces and ion drag $^{4-6}$. Heating by solar photons is insufficient at giant planets, and yet other proposed processes, such as heating by waves originating from the lower atmosphere, also fail to explain the warm equatorial temperatures $^{7}$. There remains no self-consistent explanation for measured non-auroral temperatures at present, mostly due to a lack of definitive observational constraints. Here, using high-resolution maps capable of tracing global temperature gradients at Jupiter, we show that upper-atmosphere temperatures decrease steadily from the aurora to the equator. During a period of enhanced auroral activity, likely driven by a coincident solar wind compression event, we also find a global increase in temperature accompanied by a high temperature planetary-scale structure that appears to emanate from the auroral region. These observa- 
tions indicate that Jupiter's upper atmosphere is predominantly heated via the redistribution of auroral energy.

Jupiter was recorded by the 10-metre Keck II telescope for five hours on both 14 April 2016 and 25 January 2017 using NIRSPEC (Near-InfraRed Spectrometer ${ }^{8}$ ), which was aligned northsouth along the axis of planetary rotation as shown in the guide camera image of Fig. 1a. Spectral images were acquired from light entering the slit as Jupiter rotated as shown in Fig. 1b and 1c, in which ro-vibrational emission lines of the $\mathrm{H}_{3}^{+}$ion can be seen extending from pole to equator. These ions are a major constituent of Jupiter's ionosphere and mainly reside in the $600-1000$ $\mathrm{km}$ altitude range above the 1-bar pressure surface ${ }^{9}$. The intensity ratio of two or more $\mathrm{H}_{3}^{+}$lines can be used to derive the column-averaged parameters of that ion: temperature, number density and radiance ${ }^{10,11}$. As $\mathrm{H}_{3}^{+}$has been shown to be in quasi-local thermodynamic equilibrium with Jupiter's upper atmosphere ${ }^{10,12}$, its derived temperature is representative of the region. Details of the $\mathrm{H}_{3}^{+}$fitting process and global mapping of parameters are provided in the Methods section and in Extended Data Figs. 1-2.

In the polar regions of Jupiter, electrons precipitate downward along magnetic field lines into the planet carrying an upward-directed field aligned current and giving rise to intense auroral emissions ${ }^{13}$. This magnetosphere-ionosphere coupling provides a heat source which raises local temperatures to over $1000 \mathrm{~K}^{13-15}$. In constrast, Global Circulation Models (GCMs) show that Jupiter's low latitude upper atmosphere should be $\sim 160 \mathrm{~K}$ based on solar heating ${ }^{2}$, but it is regularly observed to be over $600 \mathrm{~K}^{16}$. A potential solution is that auroral energy is redistributed from the polar regions to lower latitudes by atmospheric circulation. One GCM appeared to redistribute auroral heat successfully ${ }^{17}$, but subsequent Jupiter models have instead shown that heat within auroral is confined there by Coriolis forces and ion $\mathrm{drag}^{4-6}$. With models not converging on a clear solution at Jupiter, other hypotheses have been proposed to heat the upper atmosphere, such as the 
dissipation of acoustic waves propagated from lower altitude ${ }^{7}$

This temperature discrepancy has existed at Jupiter for nearly fifty years and has since been observed at all giant planets. It highlights a fundamental lack of understanding in this crucial atmospheric region, which governs atmospheric escape and interaction with the space environment, and has therefore been colloquially been termed the 'giant planet energy crisis' ${ }^{1,6,18}$. Models of Saturn's upper atmosphere have recently reproduced low latitude temperatures when the impact of Coriolis force is reduced by a Rayleigh drag term, which slows zonal winds and enhances meridional winds ${ }^{18}$. These results were supported by the Cassini spacecraft in latitude-altitude profiles, which found a decrease in temperature from pole to equator and found that meridional winds were strong enough to drive equatorward convection ${ }^{19}$. However, no observations or models have elucidated the mechanism by which Jupiter's low-latitude upper atmosphere is heated, and we are still missing a basic view of global temperature gradients in terms of longitude and latitude, not just at Saturn, but at any giant planet. This is particularly important since Coriolis forces and ion drag mainly act to deflect auroral winds zonally. One method of distinguishing the dominant source of heat at Jupiter's non-auroral latitudes is thus to measure the global longitude-latitude temperature gradients which, in turn, will help form a more complete understanding of energy circulation at all giant planets.

Global maps of upper-atmospheric temperature have been produced previously ${ }^{16}$, but the spatial resolution was so low that $\sim 2$ pixels covered $45-90^{\circ}$ latitude in each hemisphere, making it difficult to assess how the auroral region was connected to the rest of the planet. In Fig. 2 and 3 we show near-global maps of Jovian column averaged $\mathrm{H}_{3}^{+}$temperature, density and radiance, which are the product of several thousand individual fits to the spectral data as described in the Methods section. Using a magnetic field model, oval-shaped lines are overlain on the polar regions of Jupiter in both Fig. 2 and 3, with each representing the footprint of magnetic field lines which trace from the planet out to a particular distance in Jupiter's equatorial plane ${ }^{21}$. The main (auroral) 

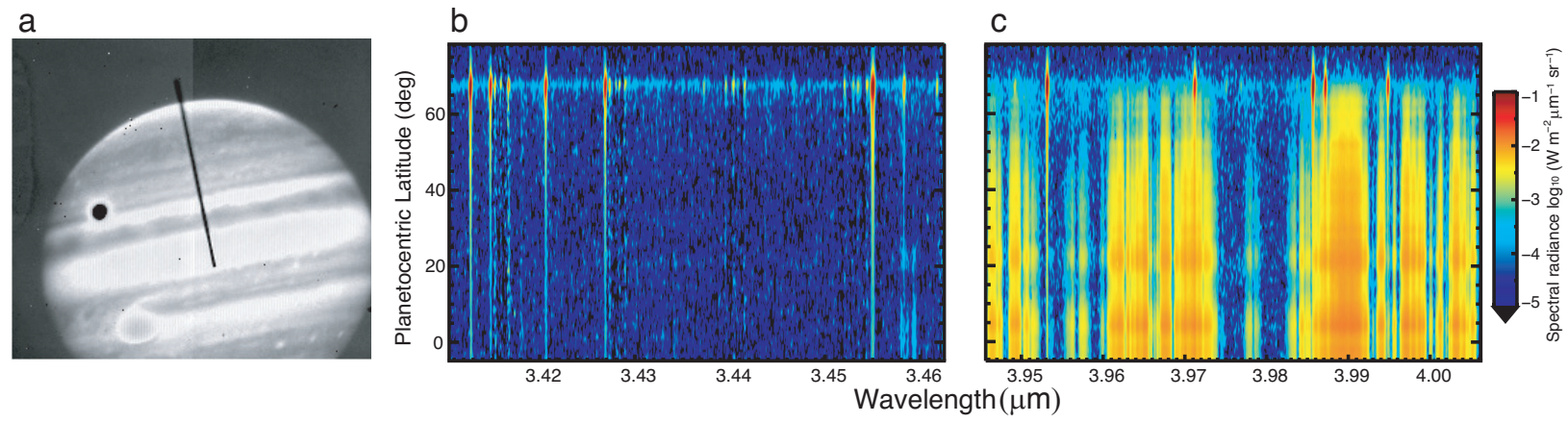

Figure 1: Example set up showing the acquisition of Jovian spectra on 14 April 2016. a, Slit-viewing Camera (SCAM) image filtered between $2.134-4.228 \mu \mathrm{m}$ wavelength. Guide images such as this are taken every 9 seconds and indicate the slit's position on the sky relative to Jupiter. In this image, the Great Red Spot (bottom left) and satellite Ganymede (top left) can be seen. $\mathbf{b}$ and $\mathbf{c}$, spectral images of Jupiter showing spectral radiance as a function of wavelength and planetocentric latitude. Most emissions seen in $\mathbf{c}$ are from the reflection of sunlight from hydrocarbons and hazes. Well-defined vertical lines are $\mathrm{H}_{3}^{+}$ro-vibrational emission lines: they are most intense in the polar regions. The $\mathrm{R}(3,0)$ and $\mathrm{Q}(1,0) \mathrm{H}_{3}^{+}$lines at $3.41277 \mu \mathrm{m}$ and $3.9529 \mu \mathrm{m}$ are the focus of this study, as their consistently high signal to noise (SNR) at all latitudes allows us to map upper-atmospheric energy balance globally. The signal to noise ratio (SNR) of $\mathrm{H}_{3}^{+}$is high at Jupiter owing to the convenient presence of a deep methane absorption band, particularly in $\mathbf{b}^{20}$. 
oval traces on average to $30 \mathrm{R}_{J}$ in Jupiter's equatorial plane ( $1 \mathrm{R}_{J}$ is Jupiter's equatorial radius of 71,492 km at the 1-bar pressure surface). The satellite footprints of Io and Amalthea are fiducial markers, mapping out from the planet to $5.9 \mathrm{R}_{J}$ and $2.54 \mathrm{R}_{J}$ in the equatorial plane, respectively.

Temperatures generally decrease from 1000 to $600 \mathrm{~K}$ between auroral latitudes and the equator, as shown by Fig. 2 and $3 . \mathrm{H}_{3}^{+}$densities, which are enhanced by aurorally-driven charged particle precipitation ${ }^{22}$, cut off sharply in the vicinity of the main oval on both dates, indicating that the direct influence of the aurora ends within several degrees of the main oval. At the same time, equatorward of the auroral regions, $\mathrm{H}_{3}^{+}$temperatures do not sharply fall with latitude. In the absence of any known sub-auroral electric current systems provided through magnetosphereionosphere coupling which cause planetary scale ion-neutral collisions, we interpret the observed temperature gradients as strong evidence that the auroral upper atmosphere is migrating away from the auroral region to lower latitudes and adjacent longitudes, transporting its heat signature along with it. This must then be facilitated principally by equatorward propagating meridional winds.

The Jovian magnetosphere, which is subjected to the solar wind, compresses in response to high solar wind dynamic pressure ${ }^{20,23}$. One model has shown that magnetospheric compression events could lead to propagation of heat away from the main auroral oval towards the equator and polar cap, introducing a temporary, local temperature increase of $50-175 \mathrm{~K}^{6,24}$. Temperatures were higher planet-wide on $25 \mathrm{Jan}$., as were main oval $\mathrm{H}_{3}^{+}$densities, so a solar wind propagation model ${ }^{25}$ was employed to examine the solar wind dynamic pressure and other parameters at Jupiter near the dates of our observations. It was found that dynamic pressures were over an order of magnitude higher within a day of the $25 \mathrm{Jan}$. observations, relative to quiet conditions, and almost three times higher compared to conditions on 14 April. This is indicated in Extended Data Figs. 4-6 (along with increased activity in other parameters). Total auroral power has previously been found to positively correlate with the duration of quiet solar wind conditions before a solar wind compression $^{23}$, so given the much longer, quieter period of solar wind activity prior to the $25 \mathrm{Jan}$. 


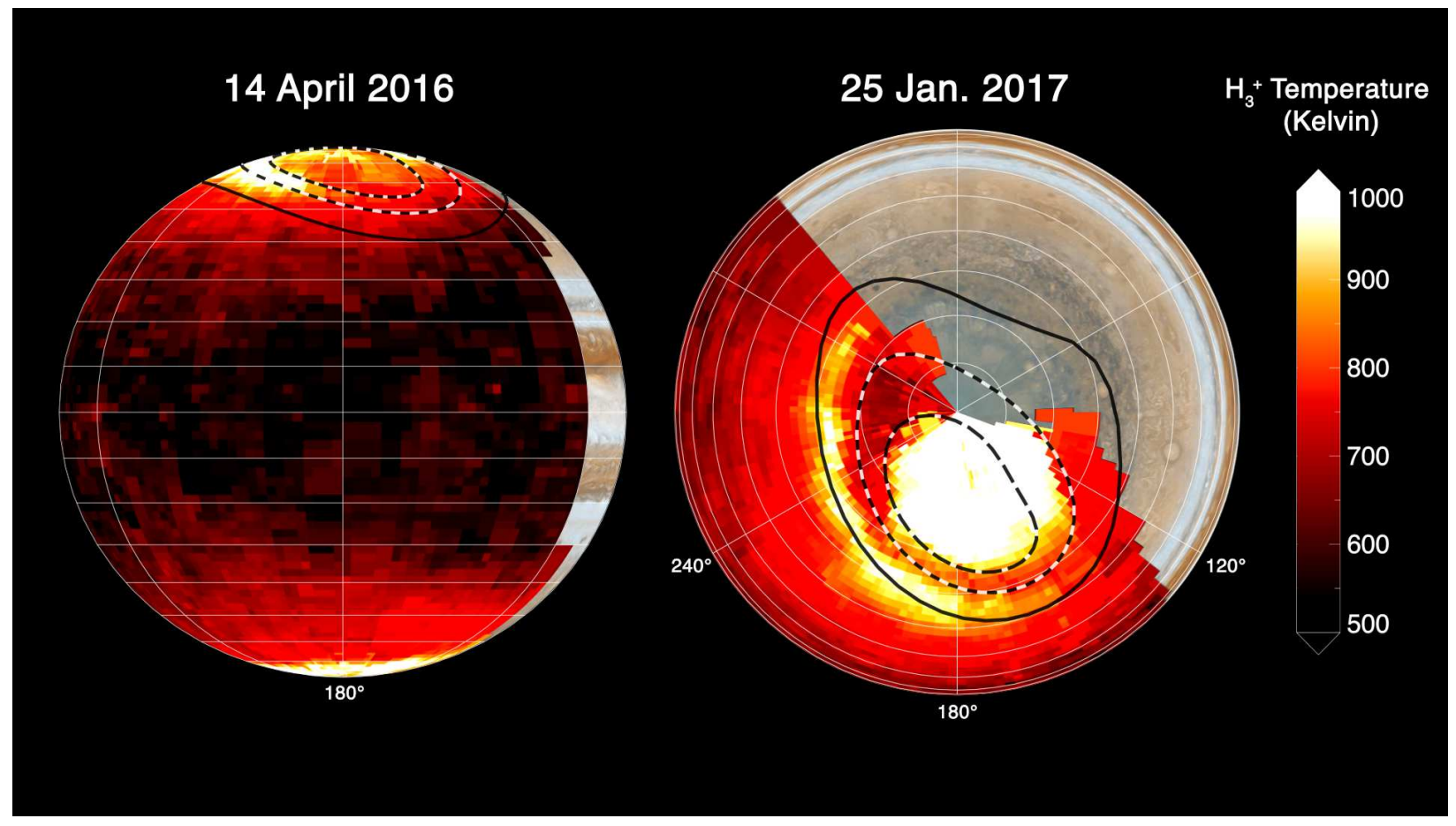

Figure 2: Orthographic projections of Jupiter's column-averaged $\mathrm{H}_{3}^{+}$temperatures on 14 April 2016 and 25 January 2017. Uncertainties in temperature are all below 5\%. Long black and white dashed lines show Jupiter's main auroral oval, short black and white dashed lines correspond to the magnetic footprint of Io and the single thick black line corresponds to the magnetic footprint of Amalthea (as described in the main text). A visible computer-generated globe of Jupiter is shown underneath the $\mathrm{H}_{3}^{+}$temperature projection. Note that Jupiter is tilted differently on each date in order to reveal different features. The longitude and latitude gridlines shown are spaced in $60^{\circ}$ and $10^{\circ}$ increments, respectively. Median (and maximum) uncertainty percentiles are 2.2\% (5\%) for 14 April 2016 and 1.6\% (5\%) for 25 Jan. 2017. The Methods section describes the mapping process and Table S1 show the spatial bin sizes that were used in each projection. 

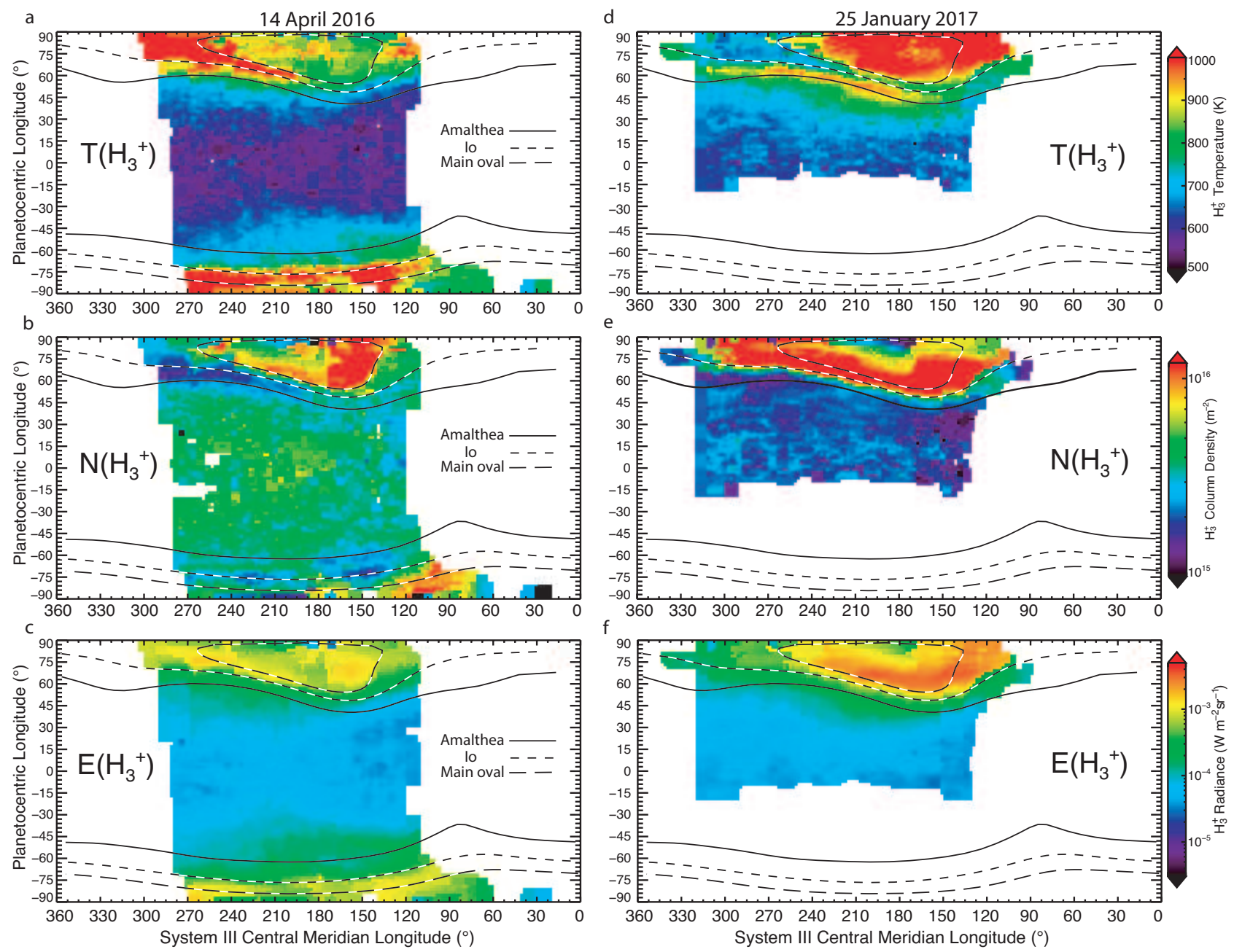

Figure 3: Equirectangular projections of Jupiter's $\mathrm{H}_{3}^{+}$column-integrated temperature, density and radiance as a function of central meridian longitude (Jovian system III) and planetocentric latitude. Temperature and radiance panels have uncertainties below 5\%, while column densities are limited to $15 \%$. Long black and white dashed lines show Jupiter's main auroral oval, short black and white dashed lines correspond to the magnetic footprint of Io and the single thick black line corresponds to the magnetic footprint of Amalthea (described in the main text). White regions indicate places in which no results met the uncertainty limited criteria. Median (and maximum) uncertainty percentiles for 14 April 2016 are: temperature 2.2\% (5\%), density 9.4\% (15\%) and radiance 2.2\% (5\%). Median (and maximum) uncertainties for $25 \mathrm{Jan} .2017$ are: temperature 1.6\% (5\%), density 5.8\% (15\%) and radiance 1.8\% (5\%). The Methods section describes the mapping process and Tables S1, S2 and S3 show the bin sizes that were used in each parameter map. 
observations reported here (relative to 14 April), we expect that auroral energy deposition was larger on 25 Jan. Factoring in the uncertainty of the modelled solar wind arrival time at Jupiter, \pm 1 days on 14 April and \pm 1.5 days on 25 Jan., we conclude that Jupiter was observed to be in the midst of a global heating event owing to solar wind compression of the Jovian magnetosphere on 25 Jan.

An unusual high temperature structure was found on 25 Jan. equatorward of the main auroral oval, extending for $160^{\circ}$ longitude and straddling the fiducial footprint of Amalthea. Here, relatively cold $\sim 800 \mathrm{~K}$ atmosphere is surrounded by hot auroral and sub-auroral atmosphere at $\sim 1000 \mathrm{~K}$. We propose that this structure is a 'snapshot' of a large region of heated upper atmosphere caught propagating equatorward away from the main auroral oval, after a 'pulse' in solar wind pressure was exerted on the magnetosphere ${ }^{6}$. An accurate calculation of the wave propagation speed is difficult as we lack knowledge of the longitudinal and latitudinal velocity vectors, so we provide a simple estimate here by assuming that the propagation is purely equatorward and following the same line of longitude. This is achieved by finding the number of degrees latitude distance between the structure's centre and the main oval, which grows with longitude and therefore with time, since the data are recorded in order of increasing longitude. Equatorward velocities for the hot feature were evaluated between $180^{\circ}$ and $260^{\circ}$ longitude in steps every $20^{\circ}$ longitude, producing a median velocity of $620 \mathrm{~ms}^{-1}$ with minima and maxima of $500 \mathrm{~ms}^{-1}$ and $1500 \mathrm{~ms}^{-1}$, respectively. These velocities are similar to equatorward-propagating travelling ionospheric disturbances observed in Earth's ionosphere $\left(300-1000 \mathrm{~ms}^{-1}\right)^{26}$ but much higher than equatorward velocites reported at Saturn (up to $\left.100 \mathrm{~ms}^{-1}\right)^{19}$ and modelled for Jupiter $\left(\sim 180 \mathrm{~ms}^{-1}\right)^{6}$.

Auroral main oval temperatures on 25 Jan. are lower than the surrounding regions, a likely indicator that $\mathrm{H}_{3}^{+}$is being produced at lower, colder altitudes by solar-wind driven enhanced charged particle precipitation ${ }^{6,27}$. The median column-integrated $\mathrm{H}_{3}^{+}$densities on 14 April and $25 \mathrm{Jan}$. were $4 \times 10^{15} \mathrm{~m}^{-2}$ and $2 \times 10^{15} \mathrm{~m}^{-2}$, respectively. The $\mathrm{F}_{10.7}$ index, an indicator of solar activity via 10.7 
cm radio emissions, was 111.8 Solar Flux Units (SFU) and 82.5 SFU on these dates, i.e. 36\% larger on 14 April, explaining in part this $\mathrm{H}_{3}^{+}$density difference. Note that retrieved $\mathrm{H}_{3}^{+}$column densities here are expected to be lower by $20 \%$ or more of their true value, owing to temperature and density gradients in the upper atmosphere ${ }^{27}$, thus differences in vertical gradients may also contribute to the measured density difference. The radiance of $\mathrm{H}_{3}^{+}$positively correlates with both $\mathrm{H}_{3}^{+}$temperature and density, showing the global ionosphere is dominated by its interactions with the magnetic field, which leaves a global imprint.

Jupiter's upper atmosphere is heated by an auroral mechanism which delivers enormous amounts of energy to the polar regions. Using global-scale measurements, we show Jovian temperatures steadily decrease from the auroral region to lower latitudes and adjacent longitudes, a result consistent with the hypothesis that energy is redistributed globally. The global circulation demonstrated here is likely replicated at all giant planets, as Jupiter's longitude-latitude gradients are consistent with temperature gradients observed (by the Cassini spacecraft) at Saturn in latitude-altitude space ${ }^{19}$. The missing heat responsible for Jupiter's energy crisis is thus predominantly auroral in nature. Coriolis and ion drag forces are unable to prevent equatorward transport of the hot polar atmosphere, perhaps due to additional Rayleigh drag caused by atmospheric waves, as recently demonstrated at $\mathrm{Saturn}^{18}$. Main auroral oval $\mathrm{H}_{3}^{+}$densities and global $\mathrm{H}_{3}^{+}$temperatures were significantly lower on 14 April compared with 25 Jan., a result consistent with solar wind model projections ${ }^{25}$ that the solar wind dynamic pressure on the Jovian magnetosphere was highest on the latter date, increasing the rates of auroral particle precipitation and global heating ${ }^{6}$. The observations on 25 Jan. also revealed, by chance, a planetary-scale-sized heated structure, which we infer is propagating away from the main auroral oval in response to a solar wind compression of the magnetosphere.

1. Strobel, D. F. \& Smith, G. R. On the temperature of the Jovian thermosphere. J. Atmos. Sci. 30, 718-725 (1973). 
2. Yelle, R. V. \& Miller, S. Jupiter's thermosphere and ionosphere, 185-218 (Cambridge University Press, 2004).

3. Smith, C. G. A., Aylward, A. D., Millward, G. H., Miller, S. \& Moore, L. E. An unexpected cooling effect in Saturn's upper atmosphere. Nature 445, 399-401 (2007).

4. Yates, J. N., Ray, L. C., Achilleos, N., Witasse, O. \& Altobelli, N. Magnetosphere-IonosphereThermosphere Coupling at Jupiter Using a Three-Dimensional Atmospheric General Circulation Model. Journal of Geophysical Research (Space Physics) 125, e26792 (2020).

5. Smith, C. G. A. \& Aylward, A. D. Coupled rotational dynamics of Jupiter's thermosphere and magnetosphere. Annales Geophysicae 27, 199-230 (2009).

6. Yates, J. N., Achilleos, N. \& Guio, P. Response of the Jovian thermosphere to a transient pulse in solar wind pressure. Planet. Space Sci. 91, 27-44 (2014).

7. O'Donoghue, J., Moore, L., Stallard, T. S. \& Melin, H. Heating of Jupiter's upper atmosphere above the Great Red Spot. Nature 536, 190-192 (2016).

8. McLean, I. S. et al. Design and development of NIRSPEC: a near-infrared echelle spectrograph for the Keck II telescope. In Fowler, A. M. (ed.) Society of Photo-Optical Instrumentation Engineers (SPIE) Conference Series, vol. 3354 of Society of Photo-Optical Instrumentation Engineers (SPIE) Conference Series, 566-578 (1998).

9. Uno, T. et al. Vertical emissivity profiles of Jupiter's northern $\mathrm{H}_{3}{ }^{+}$and $\mathrm{H}_{2}$ infrared auroras observed by Subaru/IRCS. J. Geophys. Res. 119, 10,219-10,241 (2014).

10. Melin, H. et al. On the anticorrelation between $\mathrm{H}_{3}^{+}$temperature and density in giant planet ionospheres. Mon. Not. R. Astron. Soc. 438, 1611-1617 (2014).

11. O'Donoghue, J. et al. Observations of the chemical and thermal response of 'ring rain' on Saturn's ionosphere. Icarus 322, 251-260 (2019). 
12. Miller, S., Tennyson, J., Geballe, T. R. \& Stallard, T. Thirty years of $\mathrm{H}_{3}{ }^{+}$astronomy. Reviews of Modern Physics 92, 035003 (2020).

13. Cowley, S. W. H. et al. A simple axisymmetric model of magnetosphere-ionosphere coupling currents in Jupiter's polar ionosphere. J. Geophys. Res. 110, 11209 (2005).

14. Moore, L. et al. Variability of Jupiter's IR $\mathrm{H}_{3}{ }^{+}$aurorae during Juno approach. Geophys. Res. Lett. 44, 4513-4522 (2017).

15. Johnson, R. E. et al. Mapping $\mathrm{H}_{3}{ }^{+}$Temperatures in Jupiter's Northern Auroral Ionosphere Using VLT-CRIRES. J. Geophys. Res. (Space Physics) 123, 5990-6008 (2018).

16. Lam, H. A. et al. A Baseline Spectroscopic Study of the Infrared Auroras of Jupiter. Icarus 127, 379-393 (1997).

17. Majeed, T., Waite, J. H., Bougher, S. W. \& Gladstone, G. R. Processes of equatorial thermal structure at Jupiter: An analysis of the Galileo temperature profile with a three-dimensional model. Journal of Geophysical Research (Planets) 110, E12007 (2005).

18. Müller-Wodarg, I. C. F. et al. Atmospheric Waves and Their Possible Effect on the Thermal Structure of Saturn's Thermosphere. Geophys. Res. Lett. 46, 2372-2380 (2019).

19. Brown, Z. et al. A pole-to-pole pressure-temperature map of Saturn's thermosphere from Cassini Grand Finale data. Nature Astronomy 4, 872-879 (2020).

20. Connerney, J. E. P. \& Satoh, T. The $\mathrm{H}_{3}{ }^{+}$ion: a remote diagnostic of the jovian magnetosphere. In Astronomy, physics and chemistry of $\mathrm{H}_{3}{ }^{+}$, vol. 358, 2359-2559 (2000).

21. Connerney, J. E. P. et al. A New Model of Jupiter's Magnetic Field From Juno's First Nine Orbits. Geophys. Res. Lett. 45, 2590-2596 (2018). 
22. Moore, L., Galand, M., Mueller-Wodarg, I. \& Mendillo, M. Response of Saturn's ionosphere to solar radiation: Testing parameterizations for thermal electron heating and secondary ionization processes. Planetary and Space Science 57, 1699-1705 (2009).

23. Kita, H. et al. Characteristics of solar wind control on Jovian UV auroral activity deciphered by long-term Hisaki EXCEED observations: Evidence of preconditioning of the magnetosphere? Geophys. Res. Lett. 43, 6790-6798 (2016).

24. Cowley, S. W. H. et al. A simple axisymmetric model of magnetosphere-ionosphere coupling currents in Jupiter's polar ionosphere. J. Geophys. Res. 110, 11209 (2005).

25. Tao, C., Kataoka, R., Fukunishi, H., Takahashi, Y. \& Yokoyama, T. Magnetic field variations in the jovian magnetotail induced by solar wind dynamic pressure enhancements. Journal of Geophysical Research: Space Physics 110 (2005).

26. Figueiredo, C. A. O. B. et al. Large-scale traveling ionospheric disturbances observed by GPS dTEC maps over North and South America on Saint Patrick's Day storm in 2015. Journal of Geophysical Research (Space Physics) 122, 4755-4763 (2017).

27. Moore, L. et al. Modelling $\mathrm{H}_{3}{ }^{+}$in planetary atmospheres: effects of vertical gradients on observed quantities. Philosophical Transactions of the Royal Society of London Series A 377, 20190067 (2019).

28. O'Donoghue, J. et al. Ground-based observations of Saturn's auroral ionosphere over three days: Trends in $\mathrm{H}_{3}{ }^{+}$temperature, density and emission with Saturn local time and planetary period oscillation. Icarus 263, 44-55 (2016).

29. Neale, L., Miller, S. \& Tennyson, J. Spectroscopic Properties of the $\mathrm{H}_{3}^{+}$Molecule: A New Calculated Line List. Astrophys. J. 464, 516-520 (1996). 
30. Markwardt, C. B. Non-linear Least-squares Fitting in IDL with MPFIT. In Bohlender, D. A., Durand, D. \& Dowler, P. (eds.) Astronomical Data Analysis Software and Systems XVIII, vol. 411 of Astronomical Society of the Pacific Conference Series, 251 (2009). 0902.2850.

\section{Acknowledgements}

The data presented herein were obtained at the W.M. Keck Observatory, which is operated as a scientific partnership among the California Institute of Technology, the University of California and NASA. The Observatory was made possible by the generous financial support of the W.M. Keck Foundation. The authors wish to recognize and acknowledge the significant cultural role and reverence that the summit of Maunakea has always had within the indigenous Hawaiian community. We are fortunate to have the opportunity to conduct observations from this mountain. L.M. was supported by the National Aeronautics and Space Administration (NASA) under grant no. NNX17AF14G issued through the Solar System Observations Program and grant no. 80NSSC19K0546 issued through the Solar System Workings Program.

\section{Author Contributions}

J.O'D. collected, analysed and interpreted the data and wrote the paper. L.M. assisted in collection and reduction of data and interpretation of the results. T.B. assisted in key data reduction. H.M. provided computer code necessary for the analysis of data and with T.S. both assisted in data collection, analysis and interpretation. J.E.P.C. provided code to map Jupiter's magnetic field and assisted in the interpretation of data. C.T. provided solar wind simulation results. All authors provided comments on the manuscript.

\section{Author information}

Reprints and permissions information is available at www.nature.com/reprints. The authors declare no competing financial interests: details accompany the full-text HTML version of the paper at www.nature.com/nature. Correspondence and requests for data and information should be ad- 
dressed to JO’D (jameso@ac.jaxa.jp).

Methods Additional observing details. On 14 April 2016 and 25 January 2017 Jupiter was recorded between 04:53 - 10:22 UTC and 11:36 - 16:28 UTC, respectively. The spectrometer slit measured $24^{\prime \prime}$ long by $0.432^{\prime \prime}$ wide as shown by Fig. 1 , and each pixel along the slit had a angular resolution of $0.144^{\prime \prime}$ per pixel. The spectral resolution was $\lambda / \delta \lambda \sim 25,000$. On 14 April, each of the 115 recorded spectral images of Jupiter were 30 seconds long and formed by six integrations each five seconds long, while on 25 Jan. the 80 recorded spectral images of Jupiter were 60 seconds long and formed by six integrations each ten second long. The process of saving spectral images and nodding the telescope between positions results in overhead time which led to an average elapsed time between Jupiter spectra of 2.4 minutes (14 April) and 3.4 minutes (25 Jan.), so Jupiter rotated a respective $1.4^{\circ}$ and $2.3^{\circ}$ in longitude during this time.

Absolute calibration. For the spectral images, standard astronomical data reduction techniques were applied such as the subtraction of sky spectra from Jupiter spectra to remove unwanted emissions of the Earth's atmosphere (mainly from water), and the accounting of non-uniformity in the response of the NIRSPEC detector via flat fielding and dark current subtraction. To convert the photon counts at the detector to units of physical flux a stellar flux calibration was performed using the A0 stars HR2250 and HR3314 for 14 April and 25 Jan., respectively. This process is outlined in detail in previous studies ${ }^{10,14,28}$.

Spatially mapping spectra. While the width of the slit is 0.432 arcseconds, the longitudes assigned to the slit have a wider range due to atmospheric seeing. The 14 April and 25 Jan. observation nights had clear skies with an atmospheric seeing of $0.61^{\prime \prime}$ and $0.81^{\prime \prime}$, respectively. The use of multiple guider images within each spectral image allowed for tracking errors to be accounted for, such that the derived position of the slit on Jupiter was from the average position of the slit 
seen in the guiding images. Due to the width of the slit, atmospheric seeing and the close distances on the planet between each spectral image, multiple spectra can be ascribed to a single longitude $\times$ latitude cell (spatial bin). In this work we use five spatial bin sizes: $10^{\circ} \times 10^{\circ}, 8^{\circ} \times 8^{\circ}, 6^{\circ} \times 6^{\circ}$, $4^{\circ} \times 4^{\circ}$ and $2^{\circ} \times 2^{\circ}$ longitude $\times$ latitude. All data was arranged into five 4-dimensional arrays for each observation night of dimensions longitude $\times$ latitude $\times$ spectra $\times$ overlap - the overlap dimension holds the multiple available spectra of each spatial bin, as displayed in Extended Data Fig. 1. Each of the 4-dimensional arrays was collapsed in to the 3 -dimensions longitude $\times$ latitude $\times$ spectra, by taking the median value of each available spectral element. For example, the spectral dimension has 2048 elements, and for a spatial bin which includes 50 overlapping spectra that means each of the 2048 spectral elements has 50 values associated with it. By taking the median of the 50 available values we ensure each spectral element is not skewed towards outlying data. Larger spatial bin sizes encompass more overlapping data, improving the statistical accuracy of the median value obtained, but at the cost of spatial resolution.

Fitting to $\mathbf{H}_{3}^{+}$. The $\mathrm{H}_{3}^{+}$ion has millions of rotational-vibration transition lines that vary in intensity depending on the ion temperature ${ }^{29}$, and by finding the ratio between two or more emission lines we can obtain the $\mathrm{H}_{3}^{+}$temperature. The total number of emitting ions can then be calculated by dividing the observed emission by that of a single $\mathrm{H}_{3}^{+}$ion emitting at the temperature found above, producing a line of sight column-integrated density. A cosine function correction of the planetary emission angle was applied to remove the line of sight effects of viewing geometry. The radiance of $\mathrm{H}_{3}^{+}$(also known as the $\mathrm{H}_{3}^{+}$radiative cooling rate) is then found by summing the modelled emission intensities over all wavelengths. In this work the $\mathrm{R}(3,0)$ and $\mathrm{Q}(1,0) \mathrm{H}_{3}^{+}$lines at $3.41277 \mu \mathrm{m}$ and $3.9529 \mu \mathrm{m}$ (respectively) were used due to their consistently high SNR at all latitudes. These $\mathrm{H}_{3}^{+}$ lines were fitted to and characterised using MPFIT, a least-squares curve fitting routine ${ }^{30}$, as shown in the example fits of Extended Data Fig. 2. Non- $\mathrm{H}_{3}^{+}$emissions were found at some latitudes and was subtracted. The data was then passed to a computational model which determines the parameters of $\mathrm{H}_{3}^{+}$based on the line ratios as described by the previous paragraph ${ }^{10}$. Uncertainties 
in MPFIT and the $\mathrm{H}_{3}^{+}$fitting model were propagated through and reflected in the results. Note that these observations are column integrations of the entire path-length of the ionosphere and convolve all vertical structure. Models have demonstrated that these retrieved column-integrated $\mathrm{H}_{3}^{+}$densities represent the lower limits of actual values, while column-averaged $\mathrm{H}_{3}^{+}$temperatures primarily represent mainly the temperature at the altitude peak of $\mathrm{H}_{3}^{+}$density ${ }^{27}$.

Uncertainty-limited mapping of $\mathrm{H}_{3}^{+}$parameters. The data in every spatial cell of the five data cubes were fitted so as to produce parameter maps of $\mathrm{H}_{3}^{+}$column-integrated temperature, density and radiance, along with corresponding uncertainties. A total of 15 maps were produced for each night: three $\mathrm{H}_{3}^{+}$parameters at the five aforementioned spatial bin sizes. Ideally this study would use only the $2^{\circ} \times 2^{\circ}$ maps, but these smaller bins can have lower SNR outside of the hot auroral regions and thus undesirably high uncertainties. In such a case, selecting a larger $4^{\circ} \times 4^{\circ}$ bin size to gather more signal and reduce uncertainties is preferable, even though it reduces our ability to see fine detail spatially. In order to produce a map populated by low uncertainty data at the smallest bin sizes possible planet-wide, we introduce a technique called uncertainty-limited binning. For example, an $\mathrm{H}_{3}^{+}$temperature map is produced by starting with a blank map, and then all $2^{\circ} \times 2^{\circ}$ longitude $\times$ latitude resolution temperatures that have uncertainties under $5 \%$ are added. For parts of the map which were not populated by this first pass, data is drawn from the next spatial size up - the $4^{\circ} \times 4^{\circ}$ temperature map (again with uncertainties under than $5 \%$ ) - and this process is then iterated for all remaining larger spatial bin sizes up to $10^{\circ} \times 10^{\circ} . \mathrm{H}_{3}^{+}$column-integrated temperature and radiance maps are uncertainty-limited to $5 \%$, while densities are limited to $15 \%$.

\section{Data Availability}

Observational data that are the basis of this study are publicly available on the Keck telescope observatory archive at 'https://koa.ipac.caltech.edu/cgi-bin/KOA/nph-KOAlogin?more' under Semester search terms '2016A' or '2017A' and Principle Investigator 'ODonoghue'.

\section{Code Availability}


Open-source computer code used for fitting to $\mathrm{H}_{3}^{+}$and producing temperatures, densities and radiances is available in the Python programming language at 'https://pypi.org/project/h3ppy/'. Leastsquares curve fitting routine MPFIT is referenced in the Methods section and available as part of the Interactive Data Language (IDL) suite of available programs at 'https://www.13harrisgeospatial.com/docs/mpfit.ht 


\section{Supplementary Information}

This PDF file includes:

Table T1, T2 and T3 


\section{Table S1}

\begin{tabular}{c|c|c} 
Bin Size & 14 April 2016 & 25 Jan. 2017 \\
Lon. $\times$ Lat. & \multicolumn{1}{|c}{ Cells (\% total) } & \multicolumn{1}{c}{ Cells (\% total) } \\
\hline $2^{\circ} \times 2^{\circ}$ & $5166(65.2 \%)$ & $3761(72.4 \%)$ \\
$4^{\circ} \times 4^{\circ}$ & $1477(18.6 \%)$ & $499(9.6 \%)$ \\
$6^{\circ} \times 6^{\circ}$ & $742(9.4 \%)$ & $333(6.4 \%)$ \\
$8^{\circ} \times 8^{\circ}$ & $308(3.9 \%)$ & $255(4.9 \%)$ \\
$10^{\circ} \times 10^{\circ}$ & $228(2.9 \%)$ & $348(6.7 \%)$ \\
\hline Total: & $7921(100 \%)$ & $5196(100 \%)$
\end{tabular}

Table 1: Example distribution of 5\% uncertainty-limited temperature results in Fig. 2 and Fig. 3. $\mathbf{a}$ and $\mathbf{d}$ by bin size. Each cell of Fig. 2 and 3 is of dimension $2^{\circ} \times 2^{\circ}$ longitude $\times$ latitude, with the majority of data in each of them being sourced from the $2^{\circ} \times 2^{\circ}$ bin size 


\section{Table S2}

\begin{tabular}{c|c|c} 
Bin Size & 14 April 2016 & 25 Jan. 2017 \\
Lon. $\times$ Lat. & \multicolumn{1}{|c}{ Cells (\% total) } & \multicolumn{1}{c}{ Cells (\% total) } \\
\hline $2^{\circ} \times 2^{\circ}$ & $4193(54.1 \%)$ & $3652(70.5 \%)$ \\
$4^{\circ} \times 4^{\circ}$ & $1509(19.5 \%)$ & $555(10.7 \%)$ \\
$6^{\circ} \times 6^{\circ}$ & $1201(15.5 \%)$ & $338(6.5 \%)$ \\
$8^{\circ} \times 8^{\circ}$ & $491(6.3 \%)$ & $284(5.5 \%)$ \\
$10^{\circ} \times 10^{\circ}$ & $363(4.7 \%)$ & $352(6.8 \%)$ \\
\hline Total: & $7757(100 \%)$ & $5181(100 \%)$
\end{tabular}

Table 2: Example distribution of $20 \%$ uncertainty-limited column-integrated density results in Fig. 3. $\mathbf{b}$ and $\mathbf{e}$ by bin size. Each cell of Fig. 3. is of dimension $2^{\circ} \times 2^{\circ}$ longitude $\times$ latitude, with the majority of data in each of them being sourced from the $2^{\circ} \times 2^{\circ}$ bin size

Table S3

\begin{tabular}{c|c|c} 
Bin Size & 14 April 2016 & 25 Jan. 2017 \\
Lon. $\times$ Lat. & \multicolumn{1}{|c}{ Cells (\% total) } & \multicolumn{1}{c}{ Cells (\% total) } \\
\hline $2^{\circ} \times 2^{\circ}$ & $5890(73 \%)$ & $3890(74.1 \%)$ \\
$4^{\circ} \times 4^{\circ}$ & $1049(13 \%)$ & $455(8.7 \%)$ \\
$6^{\circ} \times 6^{\circ}$ & $542(6.7 \%)$ & $321(6.1 \%)$ \\
$8^{\circ} \times 8^{\circ}$ & $283(3.5 \%)$ & $258(4.9 \%)$ \\
$10^{\circ} \times 10^{\circ}$ & $303(3.8 \%)$ & $327(6.2 \%)$ \\
\hline Total: & $8067(100 \%)$ & $5181(100 \%)$
\end{tabular}

Table 3: Example distribution of 5\% uncertainty-limited radiance results in Fig. 3. $\mathbf{c}$ and $\mathbf{f}$ by bin size. Each cell of Fig. 3. is of dimension $2^{\circ} \times 2^{\circ}$ longitude $\times$ latitude, with the majority of data in each of them being sourced from the $2^{\circ} \times 2^{\circ}$ bin size 


\section{Extended Data Figure 1}
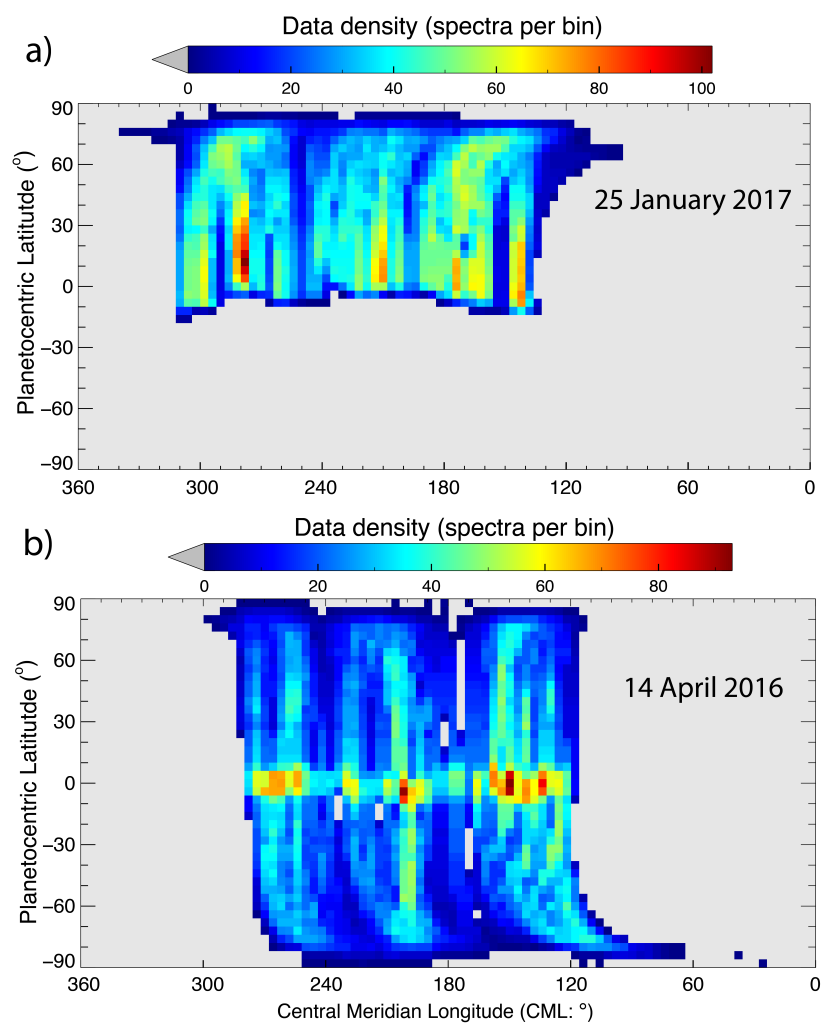

Figure 4: Data coverage (density) for a) 25 January 2017 and b) 14 April 2016 at $4^{\circ} \times 4^{\circ}$ longitude $\times$ latitude resolution. Each element contains an array of intensities as a function of wavelength as described in the main text. Data cubes like this also exist for spatial resolutions $2^{\circ} \times 2^{\circ}, 6^{\circ} \times 6^{\circ}$, $8^{\circ} \times 8^{\circ}$ and $10^{\circ} \times 10^{\circ}$. 
Extended Data Figure 2
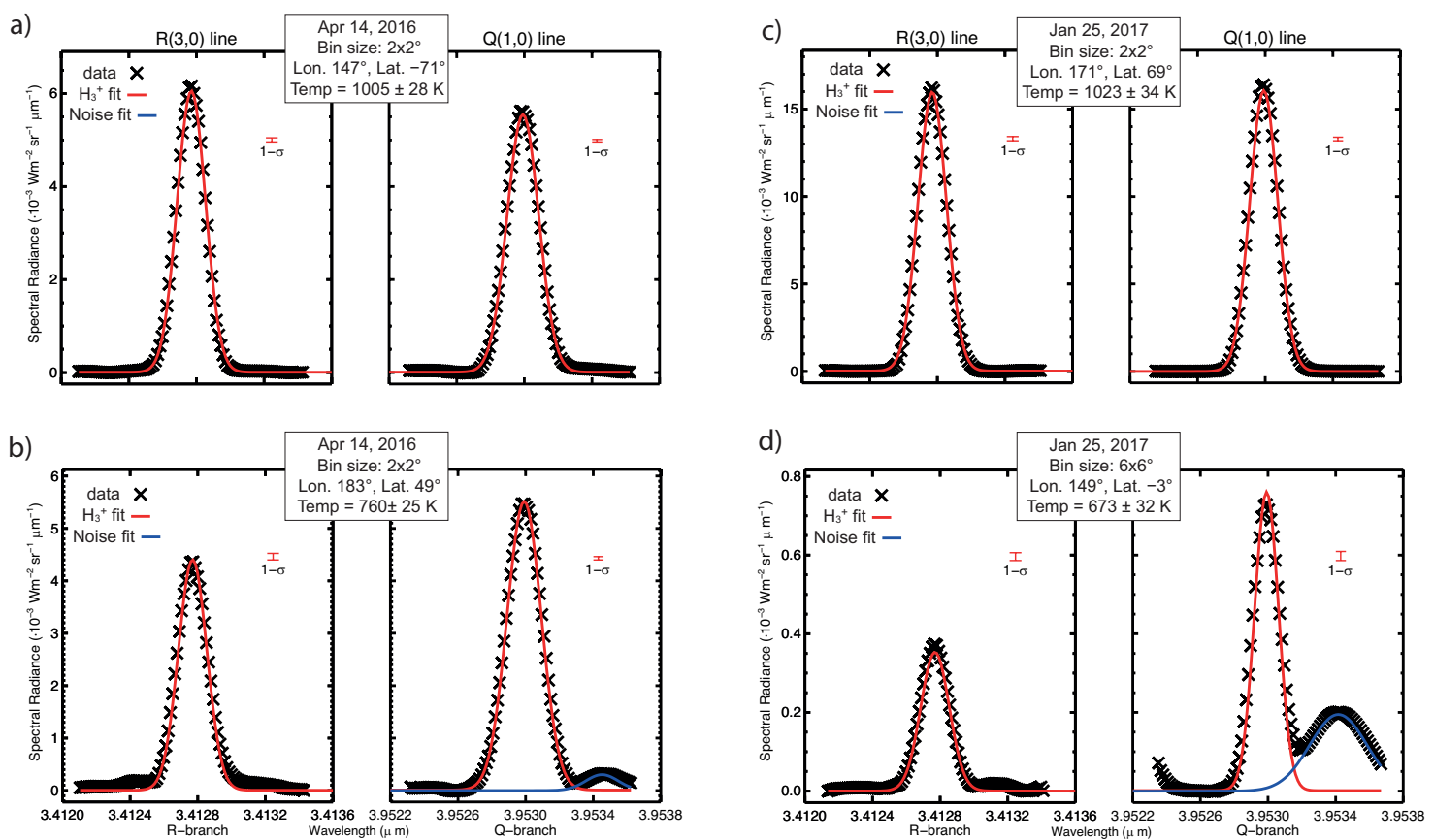

Figure 5: Selected example fits (red line) to data (black crosses) representing multiple days, differing longitudes, latitudes and spatial bin size. Fits to non- $\mathrm{H}_{3}^{+}$emissions are denoted as noise and 1 standard deviation uncertainties to each line are indicated. 


\section{Extended Data Figure 3}

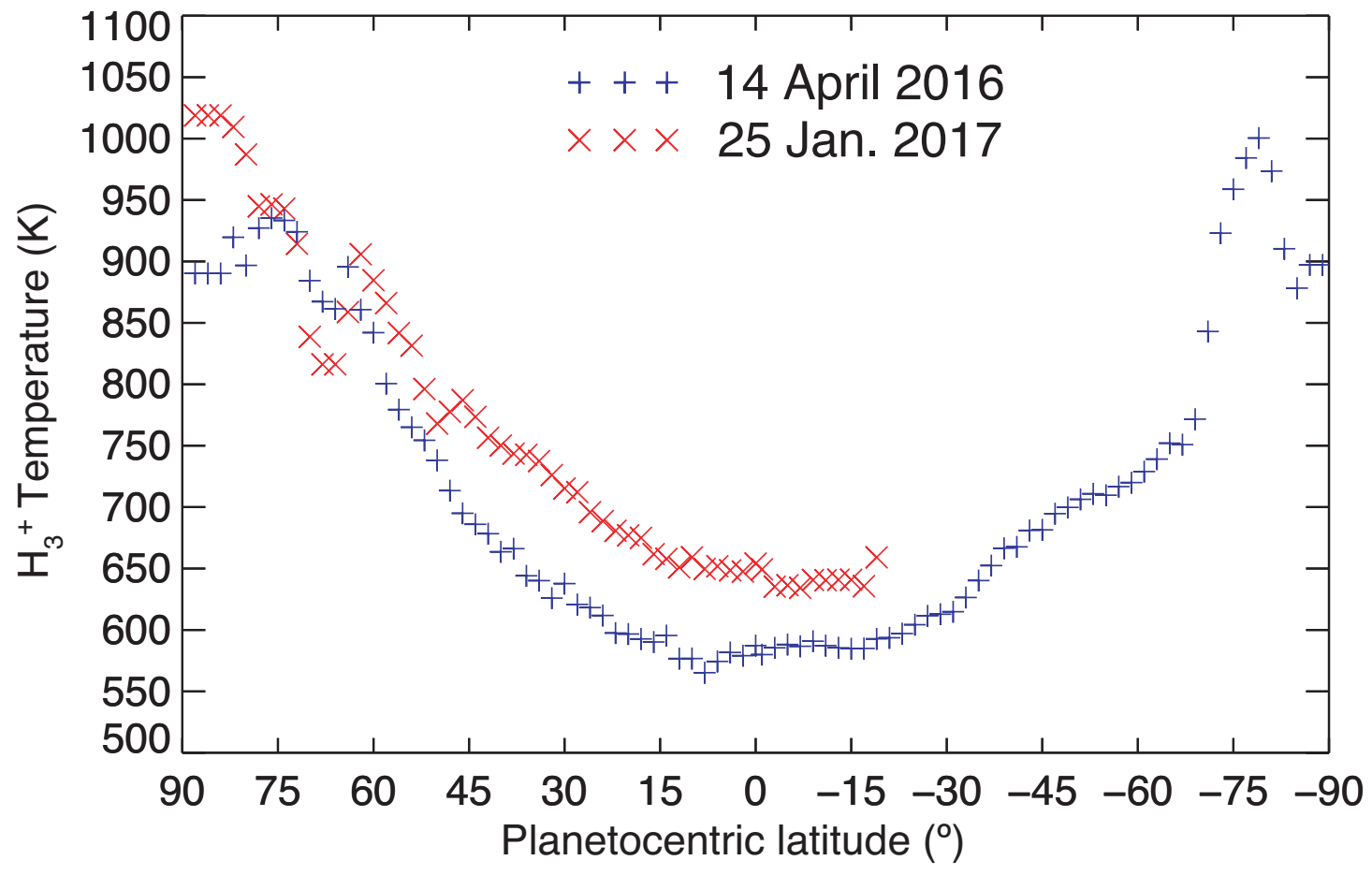

Figure 6: Jovian $\mathrm{H}_{3}^{+}$temperatures from pole to pole as a function of planetocentric latitude, based on the data in Fig. 3. Each data point is the median value for a particular latitude across the range of longitudes. 


\section{Extended Data Figure 4}

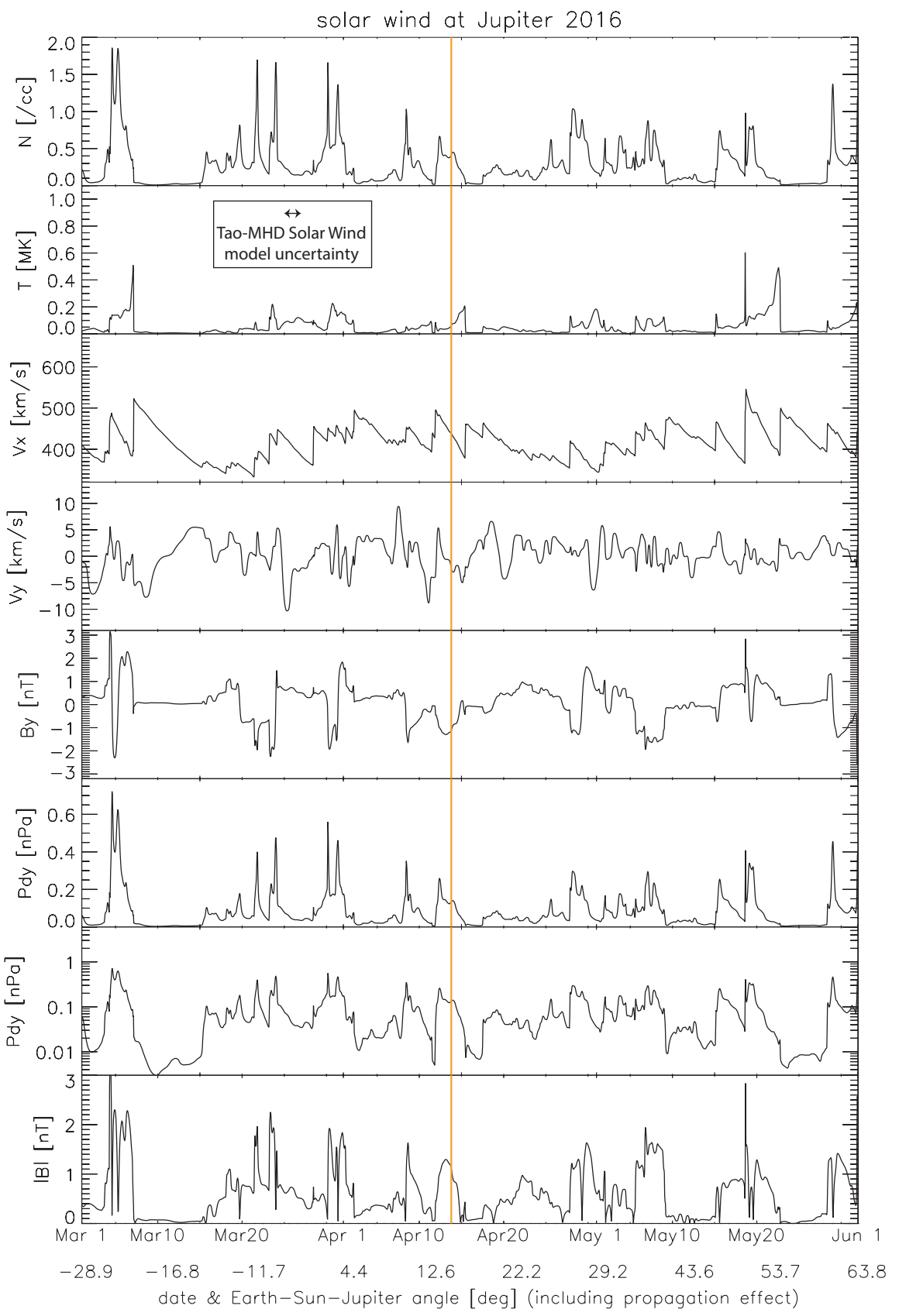

Figure 7: A 1-D magnetohydrodynamic (MHD) model of solar wind propagation ${ }^{25}$ surrounding the dates of the observations reported here. The orange shaded regions mark the period of ground-based observations. From top to bottom, each panel corresponds to solar wind density, temperature, radial (x; Jupiter-Sun line) and azimuthal (y; direction of planetary orbital motion) velocities, magnetic field $\mathrm{B}_{y}$, dynamic pressure of the solar wind plotted linearly and logarithmically and the absolute magnetic field magnitude $|\mathrm{B}|$. The 1 standard deviation uncertainty in arrival time of the solar wind at Jupiter is denoted by the horizontal, arrowed lines. 


\section{Extended Data Figure 5}

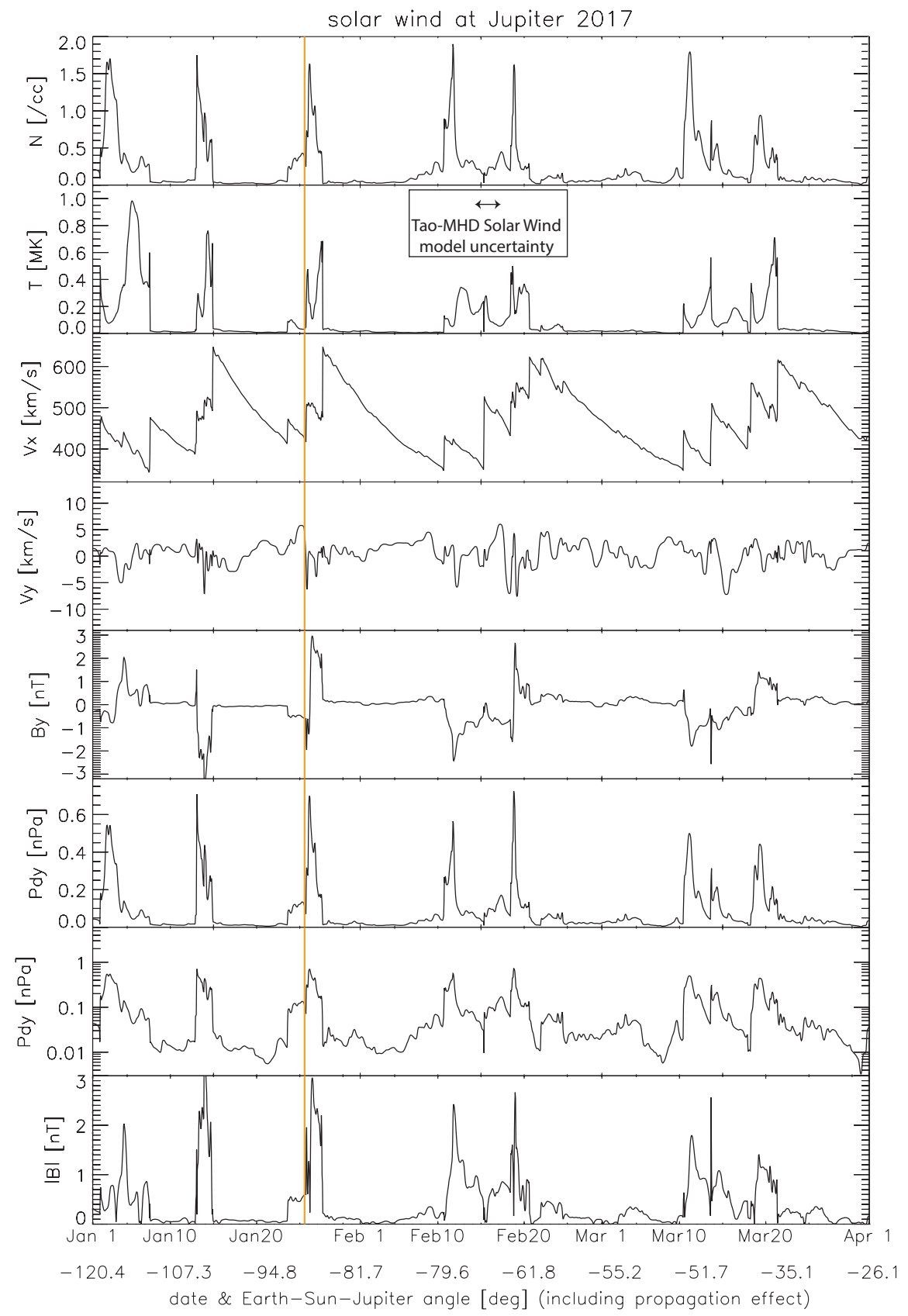

Figure 8: A 1-D magnetohydrodynamic (MHD) model of Solar wind propagation ${ }^{25}$ surrounding the dates of the observations reported here. The orange shaded regions mark the period of ground-based observations. From top to bottom, each panel corresponds to solar wind density, temperature, radial (x; Jupiter-Sun line) and azimuthal (y; direction of planetary orbital motion) velocities, magnetic field $\mathrm{B}_{y}$, dynamic pressure of the solar wind plotted linearly and logarithmically and the absolute magnetic field magnitude $|\mathrm{B}|$. The 1 standard deviation uncertainty in arrival time of the solar wind at Jupiter is denoted by the horizontal, arrowed lines. 


\section{Extended Data Figure 6}
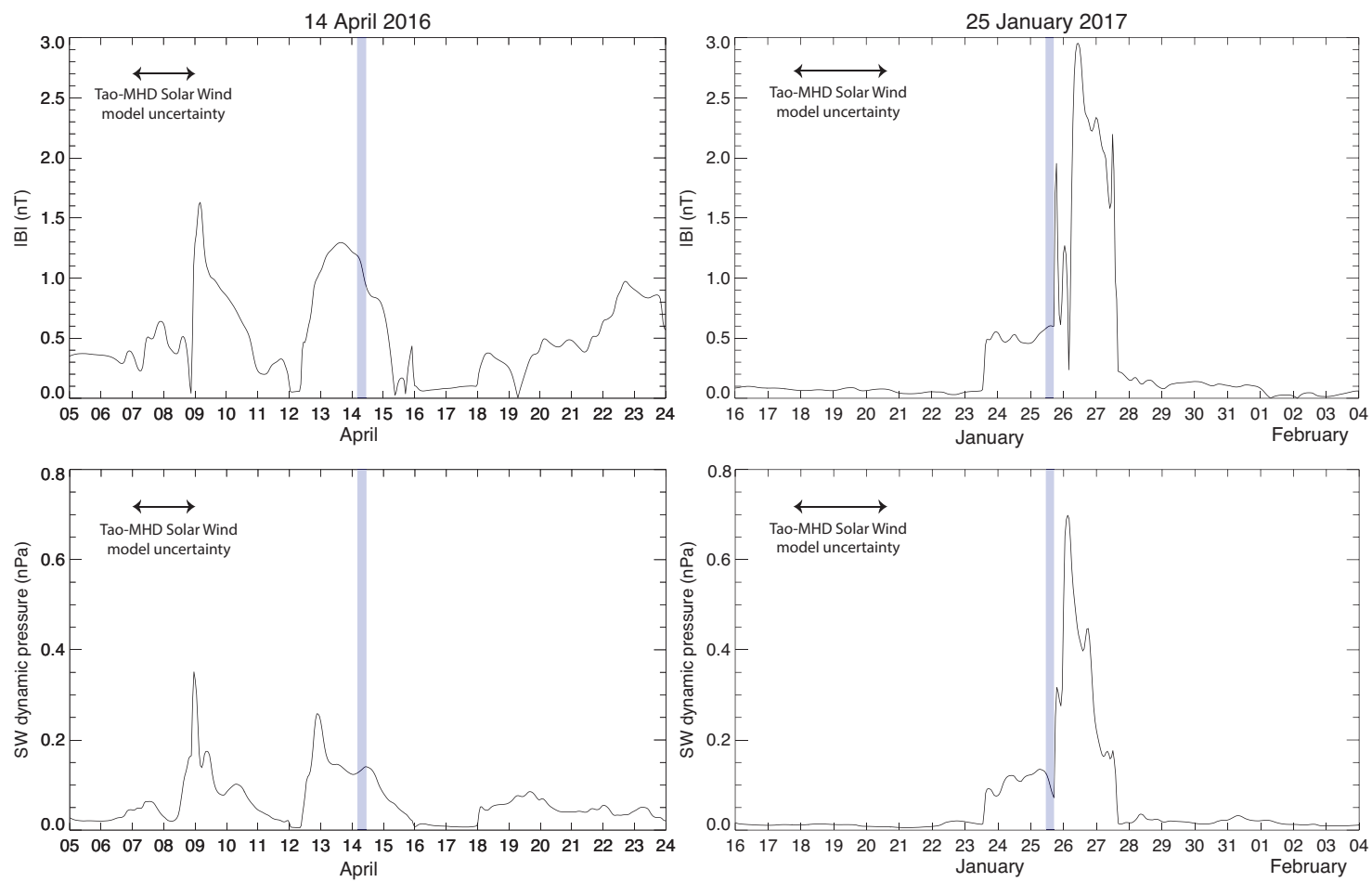

Figure 9: A 1-D magnetohydrodynamic (MHD) model of Solar wind propagation ${ }^{25}$ closely surrounding the dates of the observations reported here. The blue shaded regions mark the periods of ground-based observations. Absolute magnetic field magnitude $|\mathrm{B}|$ and dynamic pressure of the solar wind (SW) at Jupiter are shown as a function of date and time. The 1 standard deviation uncertainty in arrival time of the solar wind at Jupiter is denoted by the horizontal, arrowed lines. 


\section{Figures}

a

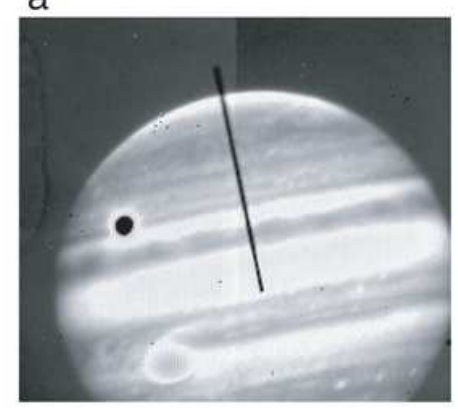

b

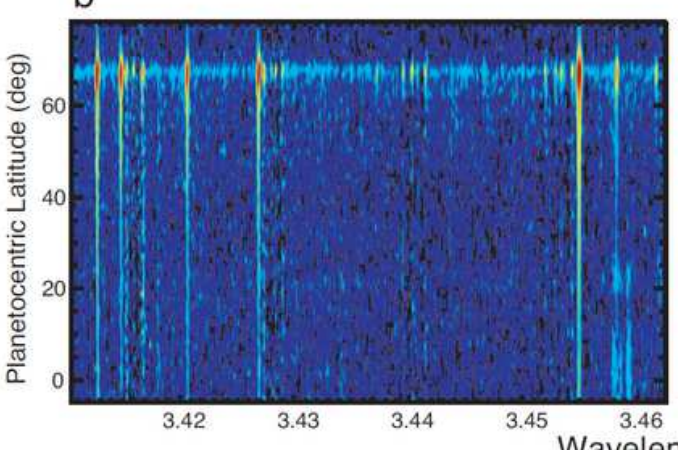

C

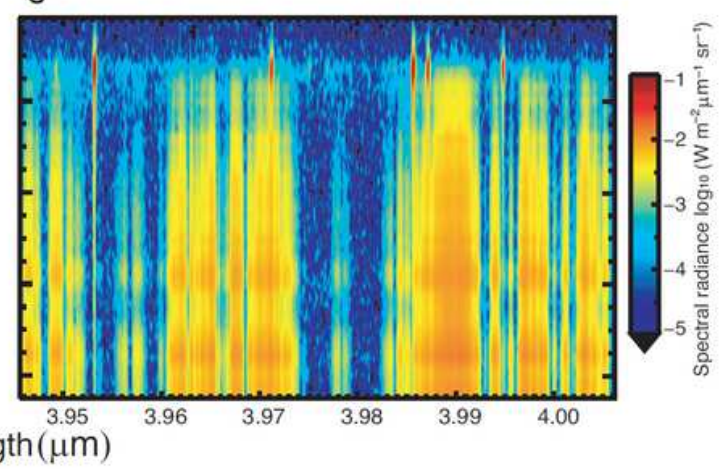

\section{Figure 1}

Example set up showing the acquisition of Jovian spectra on 14 April 2016. a, Slit-viewing Camera (SCAM) image filtered between $2.134-4.228 \mu \mathrm{m}$ wavelength. Guide images such as this are taken every 9 seconds and indicate the slit's position on the sky relative to Jupiter. In this image, the Great Red Spot (bottom left) and satellite Ganymede (top left) can be seen. b and c, spectral images of Jupiter showing spectral radiance as a function of wavelength and planetocentric latitude. Most emissions seen in $\mathrm{c}$ are from the reflection of sunlight from hydrocarbons and hazes. Well-defined vertical lines are H3 rovibrational emission lines: they are most intense in the polar regions. The $R(3,0)$ and $Q(1,0) H 3$ lines at 3.41277 $\mu \mathrm{m}$ and $3.9529 \mu \mathrm{m}$ are the focus of this study, as their consistently high signal to noise (SNR) at all latitudes allows us to map upper-atmospheric energy balance globally. The signal to noise ratio (SNR) of $\mathrm{H} 3$ is high at Jupiter owing to the convenient presence of a deep methane absorption band, particularly in b20. 


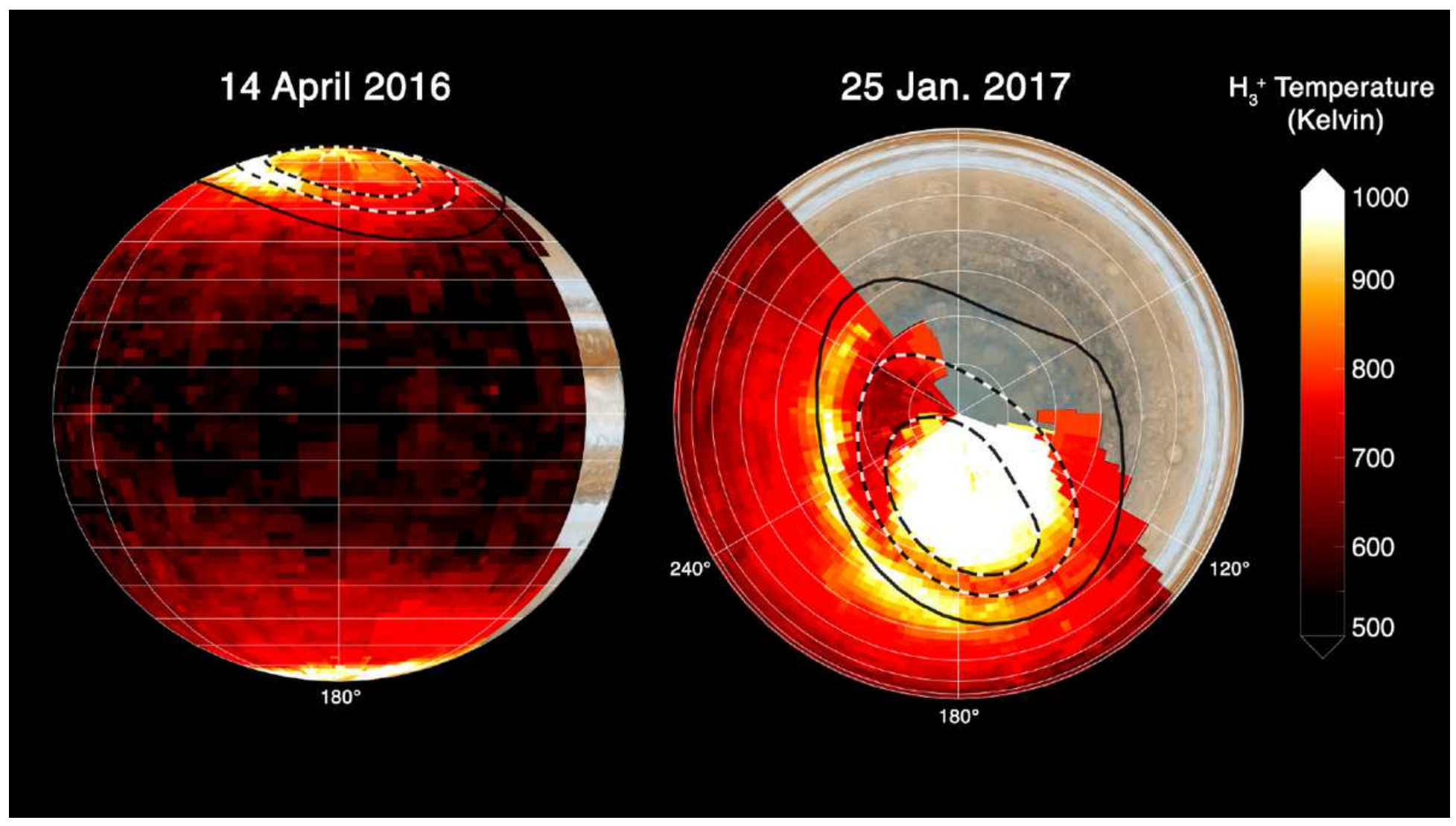

\section{Figure 2}

Orthographic projections of Jupiter's column-averaged H3 temperatures on 14 April 2016 and 25 January 2017. Uncertainties in temperature are all below 5\%. Long black and white dashed lines show Jupiter's main auroral oval, short black and white dashed lines correspond to the magnetic footprint of lo and the single thick black line corresponds to the magnetic footprint of Amalthea (as described in the main text). A visible computer-generated globe of Jupiter is shown underneath the $\mathrm{H} 3$ temperature projection. Note that Jupiter is tilted differently on each date in order to reveal different features. The longitude and latitude gridlines shown are spaced in 60囚 and 10区 increments, respectively. Median (and maximum) uncertainty percentiles are $2.2 \%$ (5\%) for 14 April 2016 and 1.6\% (5\%) for 25 Jan. 2017. The Methods section describes the mapping process and Table S1 show the spatial bin sizes that were used in each projection. 

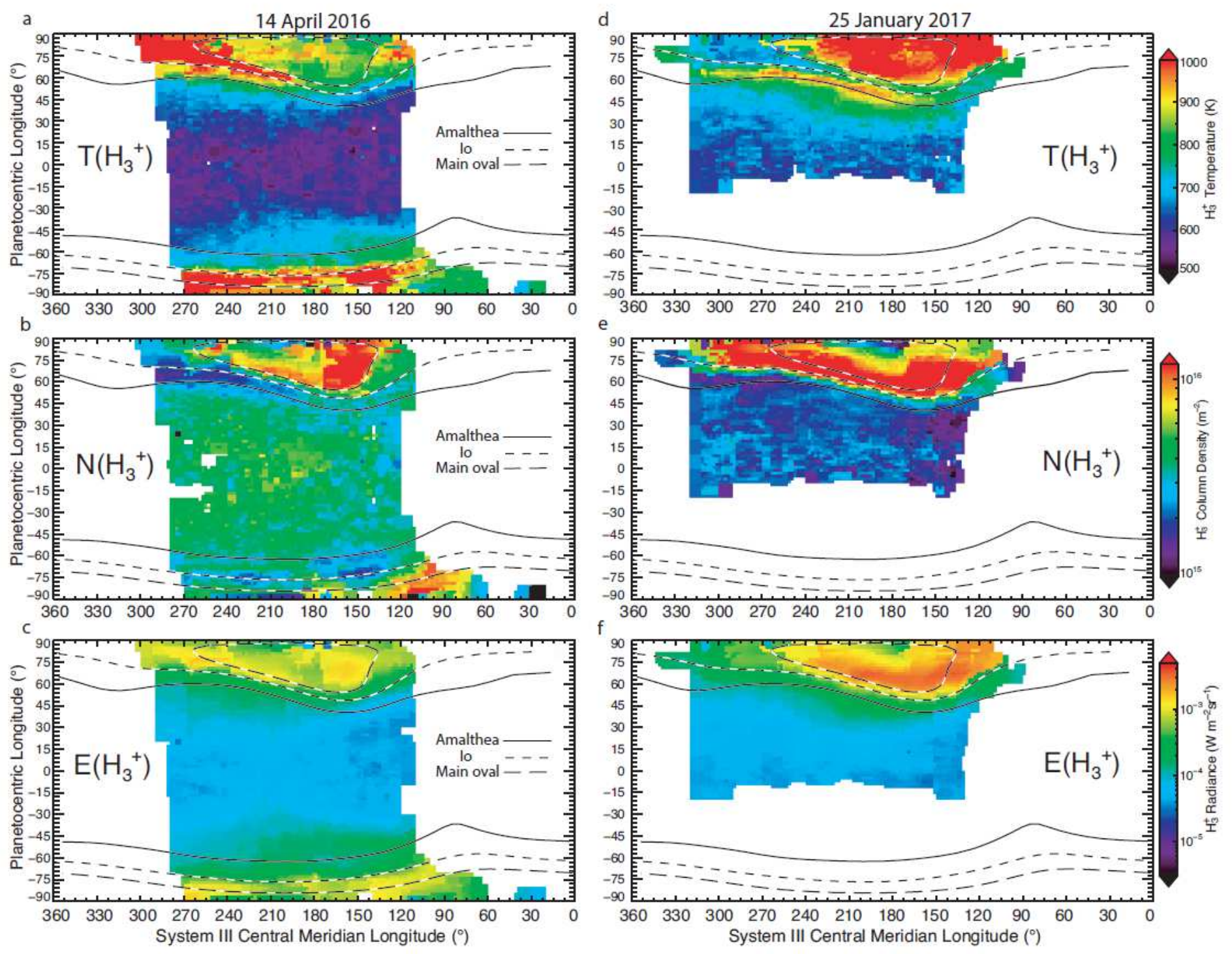

\section{Figure 3}

Equirectangular projections of Jupiter's H3 column-integrated temperature, density and radiance as a function of central meridian longitude (Jovian system III) and planetocentric latitude. Temperature and radiance panels have uncertainties below $5 \%$, while column densities are limited to $15 \%$. Long black and white dashed lines show Jupiter's main auroral oval, short black and white dashed lines correspond to the magnetic footprint of lo and the single thick black line corresponds to the magnetic footprint of Amalthea (described in the main text). White regions indicate places in which no results met the uncertainty limited criteria. Median (and maximum) uncertainty percentiles for 14 April 2016 are: temperature $2.2 \%$ (5\%), density $9.4 \%$ (15\%) and radiance 2.2\% (5\%). Median (and maximum) uncertainties for 25 Jan. 2017 are: temperature $1.6 \%(5 \%)$, density $5.8 \%(15 \%)$ and radiance $1.8 \%(5 \%)$. The Methods section describes the mapping process and Tables S1, S2 and S3 show the bin sizes that were used in each parameter map. 